\title{
A New Hybrid Filter Approach for Image Processing
}

\author{
Bekir Aksoy ${ }^{1}$, iD Osamah Khaled Musleh Salman² \\ ${ }^{1}$ Corresponding Author; Department of Mechatronics Engineering, Isparta University of Applied Sciences \\ Faculty of Technology, Isparta, TURKEY; bekiraksoy@isparta.edu.tr; 05536422737 \\ ${ }^{2}$ Department of Mechatronics Engineering, Isparta University of Applied Sciences Faculty of Technology, \\ Isparta, TURKEY; 1930654007@stud.sdu.edu.tr
}

Received 26 August 2020; Revised 5 December 2020; Accepted 10 December 2020; Published online 30 December 2020

\begin{abstract}
Today, with the rapidly advancing technology, the importance of image processing techniques is increasing. Image processing is used in many areas from facial recognition to plant disease identification. One of the important image processing stages is the filtering stage used for smoothing images and object detection. Among these filtering techniques, basic filtering techniques such as mean, median and Gaussian are used in image processing. However, these filtering techniques are known to be insufficient to achieve the desired results in some cases. In this study, a new hybrid filtering approach named Mean-Median-Gaussian (MMG) is presented using these three basic filtering techniques. It has been demonstrated that the obtained MMG hybrid algorithm gives more successful results than these three basic filtering techniques in smoothing the images and determining the boundary lines.
\end{abstract}

Keywords: Image Processing, Filters, smoothing methods, Drawing Contour, MMG

\section{Introduction}

Image processing is a method used to extract useful information from the image by converting the recorded image to digital form and then processing it [1, 2]. Image processing takes place electronically using computers, software and various algorithmic approaches [3]. Image processing involves three steps [4]. The first step is to transfer the image to electronic media with various devices such as an optical scanner and digital photography. In the second step, the transmitted image is improved, analysed and processed to reduce the noise. The last step is the output step where the image is ready for use [5].

The concept of image processing first began with the digitization of newspaper images sent by the submarine cable in the 1920s [6]. In a space conference in 1961, the idea of digital light-sensing was proposed and a panel with the proposed logic was produced in 1968. In 1975, the first digital camera was invented by Steven Sasson [7]. The majority of the research done in the field of digital image processing was carried out in the 1960s at the Jet Propulsion Laboratory, Massachusetts Institute of Technology, Bell Labs and the University of Maryland. With the development of technologies, the potential in the field of digital images has also increased.

The image used in image processing must first go through the image pre-processing stage. Image preprocessing is a step that directly affects the performance and quality of the process [8]. In the image preprocessing phase, many different techniques are applied to improve the image for reducing the noise and noise ratio [9]. One of the most used techniques in the image pre-processing stage is filtering processes. In the filtering process, it is possible to interpret the image more easily by enabling the distinction between physical properties to be clarified or eliminated thanks to the different effects given to the image $[10,11]$. Image smoothing filters are used to minimize colour differences in the image and highlight sparse structures [12]. Smoothing is an essential process for many computer vision applications [13]. In algorithms with image smoothing, gradient size is generally used as a hint [14].

In this study, a new hybrid filtering approach named Mean Median Gaussian (MMG), which is the combination of mean, median and Guassian filtering methods is presented. In this filtering approach, a new hybrid filtering technique named MMG is presented by using median, mean and Guassian basic smoothing filtering techniques. These four filters were applied to the same image and their performance was compared to show that the performance of the hybrid filtering technique was more successful than the other three filtering techniques. 
Aksoy et. al

\section{Materials and Methods}

It was stated that the MMG hybrid filtering technique used in this study was created by the combination of mean, median and Guassian filtering techniques. For this reason, detailed information about mean, median and Guassian filtering techniques is given below. In the method section, the working method of the MMG hybrid algorithm is explained in detail.

\subsection{Materials}

The structures of mean, median and Guassian filtering methods that form the structure of the MMG hybrid filter are examined in detail in this section.

\subsubsection{Median Filter}

Median filtering is a nonlinear smoothing filter and is used to remove unwanted noise in the image [15]. First, a kernel matrix is created on the image so that smoothing can be performed with the median filter. The pixel value in the centre of the kernel is replaced by the median pixel value obtained by sorting the density values of the kernel pixels neighbouring to the central pixel. Thus, with the median filtering process, the noise-containing pixels are replaced by the median value of the neighbours. The mathematical expression of the median value calculation applied to the pixels is given in Equation 1 [16].

$$
\operatorname{Median}(M)=\operatorname{Med}\left\{M_{i}\right\}\left\{\begin{array}{ll}
M_{i}(k+1) / 2 & k \text { is odd } \\
1 / 2\left[M_{i}(k / 2)+M_{i}(k / 2)+1\right] & k \text { is even }
\end{array}\right\}
$$

In Equation $1, M_{1}, M_{2}, M_{3}, \ldots, M_{k}$ is the index of neighboring pixels. Before filtering, the pixels in the image are sorted and the median value is selected. The median filter may be insufficient to remove highdensity impulse noises. For this reason, with the development of the standard median filter (SMF) method; different types of the median filter are proposed, such as the adaptive median filter (AMF), the weighted median filter (WMF), the adaptive weighted median filter (AWMF), the fast and efficient median filter (FEMF), and the noise adaptive fuzzy switching median filter (NAFSMF) [17]. AMF determines the window size based on the noise intensity for identifying and removing defective pixels. Determining the size of the filtering window greatly affects the filtering performance [18]. WMF suppresses noise intensity, noise reduction and image restoration by preserving the details of the image. AWMF defines a pixel as noise if its level is not between the maximum and minimum grey levels suitable for the filtering window. The pixel defined as noise is replaced with the average of the normal pixels in the filtering window. If the filtering window does not have a normal pixel, AWMF increases the window size [19]. FEMF uses previous information to get the original pixels in the restoration process. It detects noises quickly, intuitively, without iteration, and only improves the pixels that contain noise, without changing other pixels. NAFSMF uses histogram of the distorted image to identify noise pixels. With the obtained information, it performs fuzzy reasoning to eliminate the uncertainty caused by noise [17].

\subsubsection{Mean Filter}

Mean filter is a sliding window based spatial filtering method used for image smoothing and reducing or eliminating noise in the image. In the mean filter method, the value of the centre pixel in the window is found by taking the average of the neighbouring pixel values. The window size determines the number of neighbour pixels to be averaged. The mathematical equation given in equation 2 is used for the spatial filtering response at any $(i, j)$ point of the image by shifting the window on neighbor pixels [20].

$$
\mu[i, j]=\frac{1}{M} \sum_{k=i=1}^{i+1} \sum_{i=j=1}^{j+1} f[k, l]
$$


The $M$ value in the equation refers to the number of pixels used in the calculation, the $k$ and $l$ values represent the location of these pixels.

\subsubsection{Gaussian (Smoothing / Blurring) Filter}

The Gaussian filter is a low-pass filter commonly used in image processing applications to remove detail and noise in the image. Using the Gaussian filter, smoothing and blurring are applied on the image to remove the noise and improve the image quality [21-22]. With the Gaussian filtering method, various image/video processing applications such as edge detection, image blurring and mosaicization are easily performed [21]. The general mathematical expression of the Gaussian filter is given in Equation 3, and the mathematical expression of the Gaussian filter for two-dimensional images is given in Equation 4 [23].

$$
\begin{gathered}
G(x)=\frac{1}{\sqrt{2 \pi \sigma^{2}}} e^{-\frac{x^{2}}{2 \sigma^{2}}} \\
G(x, y)=\frac{1}{2 \pi \sigma^{2}} e^{-\frac{x^{2}+y^{2}}{2 \sigma^{2}}}
\end{gathered}
$$

where $G(x, y)$ represents gaussian filter value, $x$ and $y$ represent row and columns and $\sigma$ is the standard deviation of the Guassian distribution. Standard deviation plays an important role in the behaviour of the Gaussian function [23].

\subsection{Methods}

In this study, the method shown in Figure 1 was used. First, a kernel matrix size was determined by using a sample image. In the method part of the study, a 3x3 kernel matrix is used as an example. With this kernel, matrix size mean, median and Guassian filters were applied on the sample image. The resultant vectors of the same pixels from the images which mean, median and Guassian filtered are applied were calculated and a single image was obtained. The normalization process on the image obtained by taking the resultant vectors was done using the mathematical equation given in equation 5 . Using this equation, the normalization was performed by calculating the resultant vector of the pixel values corresponding to the three filtering methods.

$$
M M G_{i, j}=255 \cdot \frac{\sqrt{\left(\operatorname{Mean}_{i, j}\right)^{2}+\left(\operatorname{Median}_{i, j}\right)^{2}+\left(\operatorname{Gaussian}_{i, j}\right)^{2}}}{\operatorname{Max}\left(M M G_{i, j}\right)}
$$

In the equation, $M M G$ represents the new image that has been softened by filter and $\operatorname{Max}\left(M M G_{i, j}\right)$ represents the maximum pixel value obtained from the resultant vector before normalization. The results obtained using a sample image to test the performance of the MMG hybrid filter are given in the research findings section.

\section{Research Findings}

The performance of the MMG filter used in the study was evaluated according to the histogram and edge detection/segmentation image processing methods and the following findings were obtained. Firstly, mean, median and Guassian filtering methods with 5x5 kernel matrix were used on an image for smoothing and noise removal processes. The results of these transactions are given in Figure 2. 
Aksoy et. al

Original Image

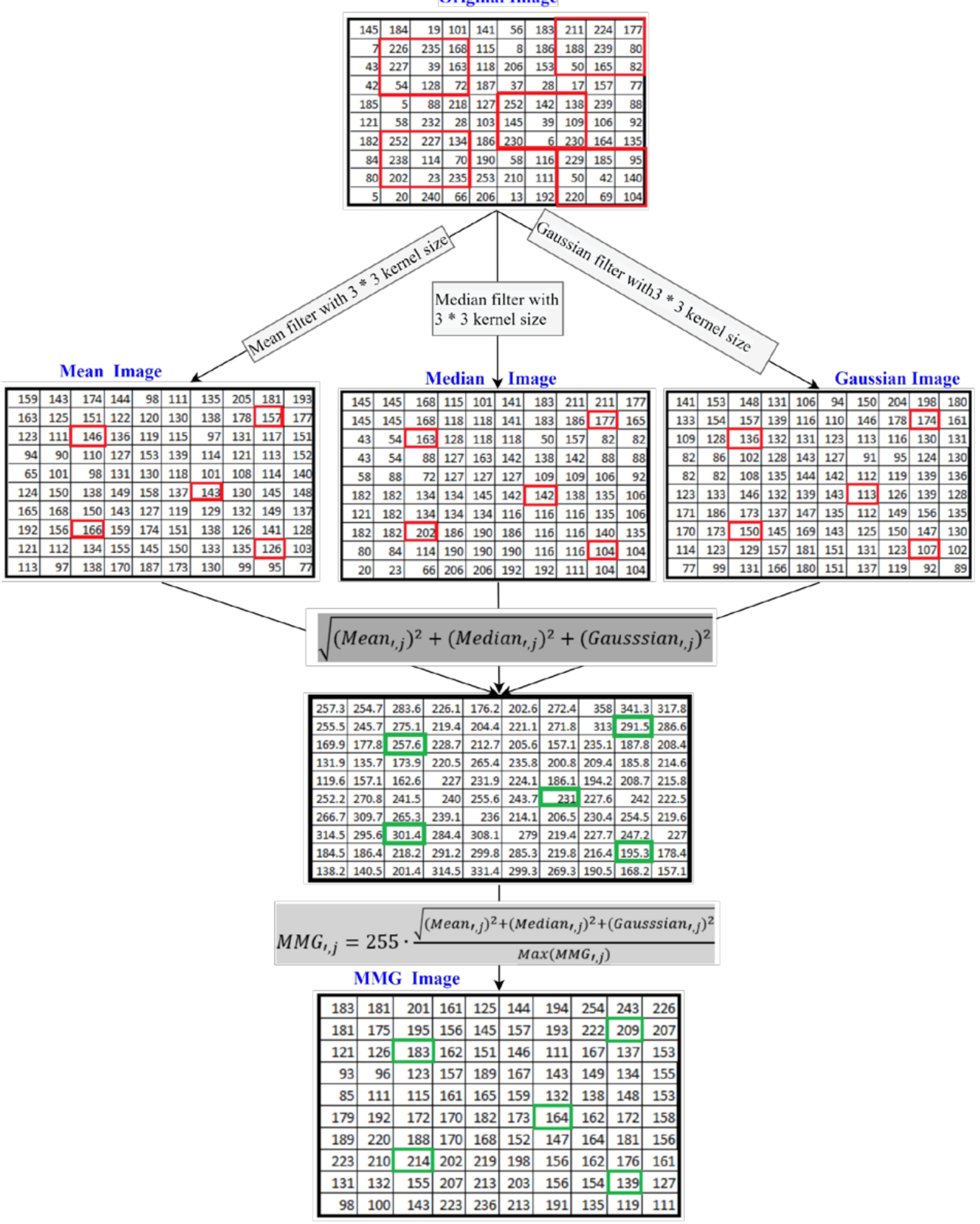

Figure 1 MMG hybrid filter structure 


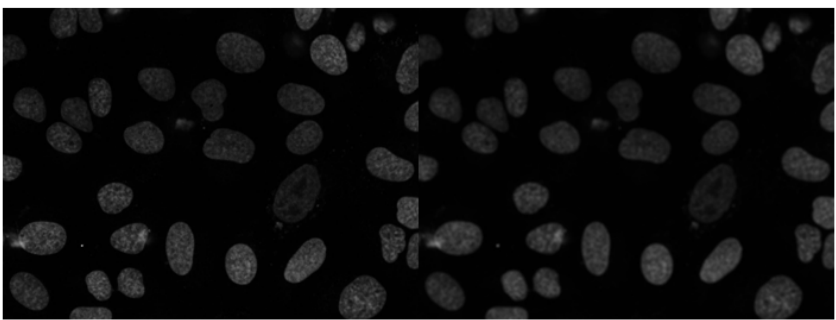

(a)

(b)

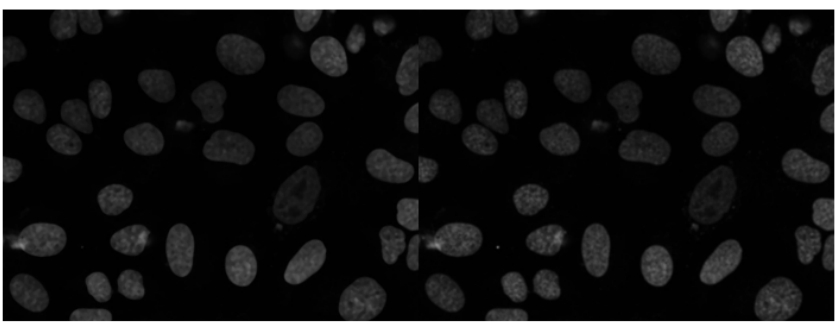

(c)

(d)

Figure 2 (a) original image, (b) mean filter applied image (c) median filter applied to image (d) Guassian filter applied image

When the images in Figure 2 are examined, it is seen that the results obtained from three different filter methods applied to the sample image are close to each other. Histogram curves of mean, median and Gaussian filters are given in Figure 3.

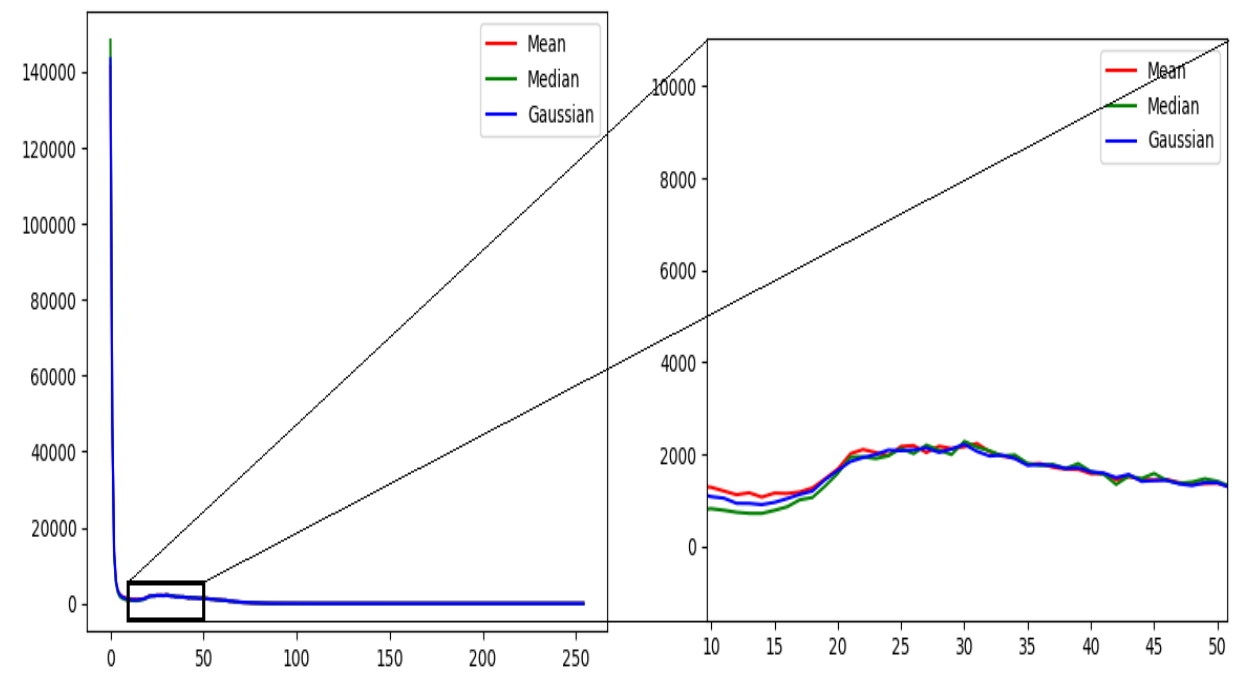

(a)

(b)

Figure 3 (a) Histogram curve of mean median and Guassian filters (b) Enlarged view of the 10 to 50-pixel range of histogram curve of mean, median and Guassian filters

The histogram curve of the entire image is given in Figure 3 (a). However, due to a large number of black pixels, a sample area with a change on the histogram curve is enlarged in order to examine the graphic in detail and enlarged image is given in Figure 3 (b). When Figure 3 (b) is examined, it is seen that mean, median and Guassian filtering methods give similar results. It is seen that the applied mean, median and Guassian filtering methods do not achieve the desired smoothing result on the image.

To obtain the desired softening results, image softening was performed using the mathematical model given in equation 5 in MMG hybrid filtering method. In Figure 4, the image obtained from MMG hybrid filter is given. 


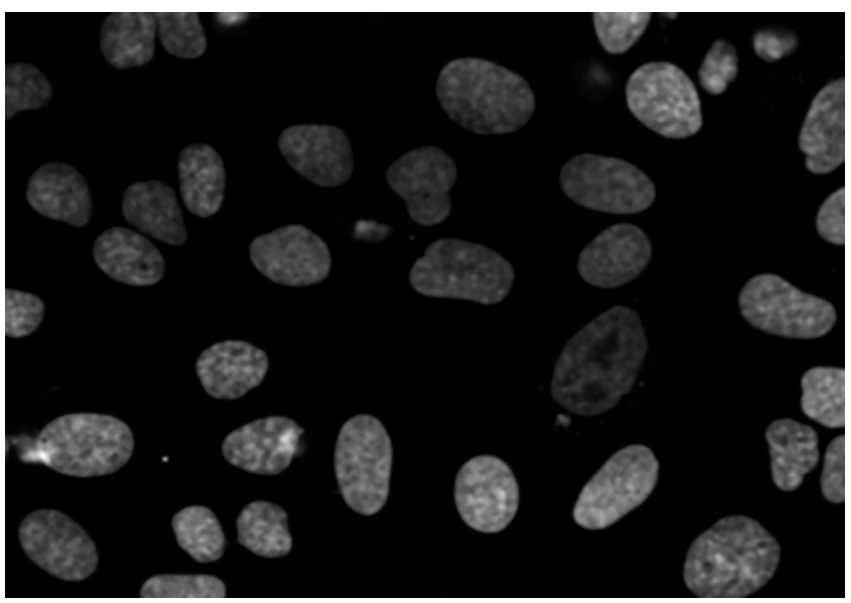

Figure 4 MMG filter applied image

When Figure 4 is examined, it is seen that the results obtained from MMG hybrid filter are more successful than the results obtained from mean, median and Guassian filters in Figure 2. It is seen that the results obtained from the MMG hybrid filter are more pronounced and there is a sharp transition between the pixels compared to the results obtained from the other three filtering methods. For example, the values of the black or white pixels in the MMG hybrid filtered image appear to be sharper than the black or white pixel values obtained in the other three filtering methods. In Figure 5, histogram curves of mean, median, Guassian and MMG filters are given.

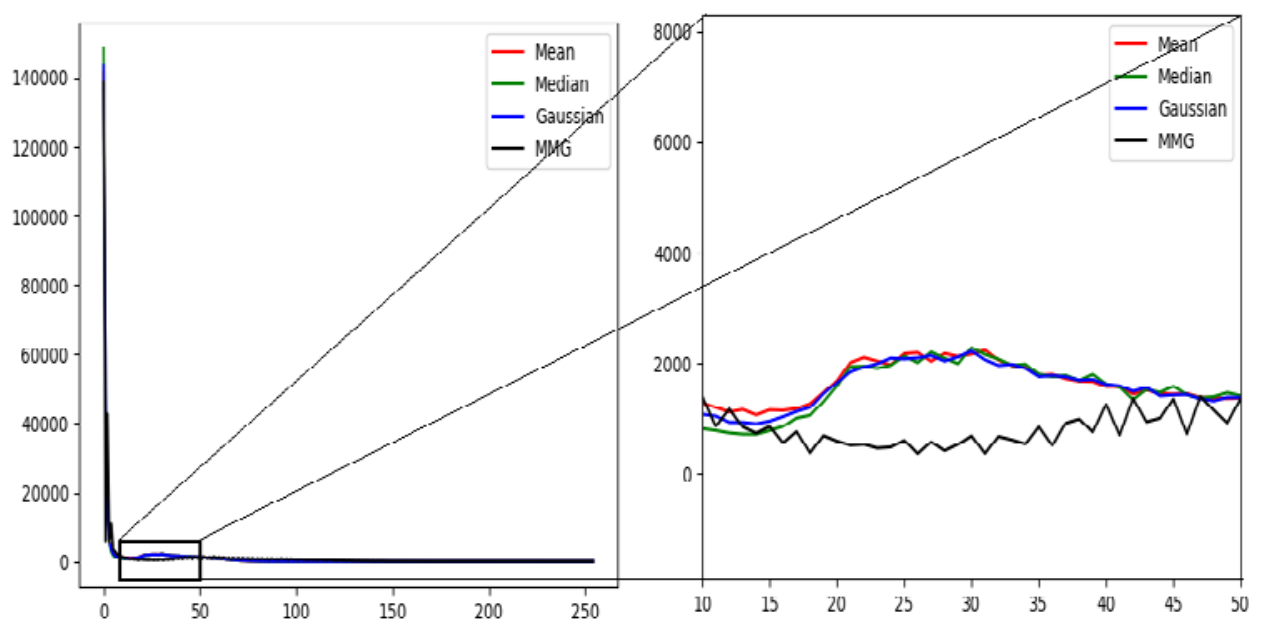

(a)

(b)

Figure 5 (a) Histogram curve of mean, median, Guassian and MMG filters (b) Enlarged view of the 10 to 50pixel range of histogram curve of mean, median, Guassian and MMG filters.

The histogram curve of the entire image is given in Figure 5 (a). However, due to the large number of black pixels, a sample area with a change on the histogram curve is enlarged in order to examine the graphic in detail and enlarged image is given in Figure 5 (b). As mentioned before in Figure 5 (b), it is seen that mean, median and Guassian filtering methods give similar results. However, in the results obtained with MMG filter, it is seen that pixel distribution is more suitable. Since there is a sharper change between pixel values in MMG filtering method, it is thought that this method will give more successful results in image processing methods such as edge detection, segmentation and object detection.

MMG filtering method was used to determine the edges on the image and the following findings were obtained about its performance. First of all, using the $5 \times 5$ kernel matrix, object edge detection was performed on the image with mean, median, Guassian and MMG filters. The results of these processes are given in figure 6 . 


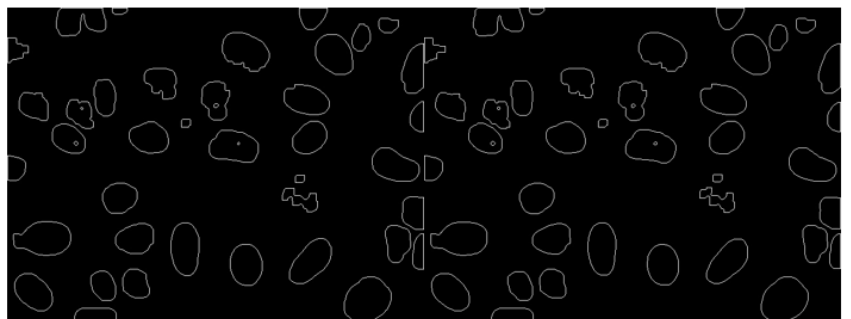

(a)

(b)

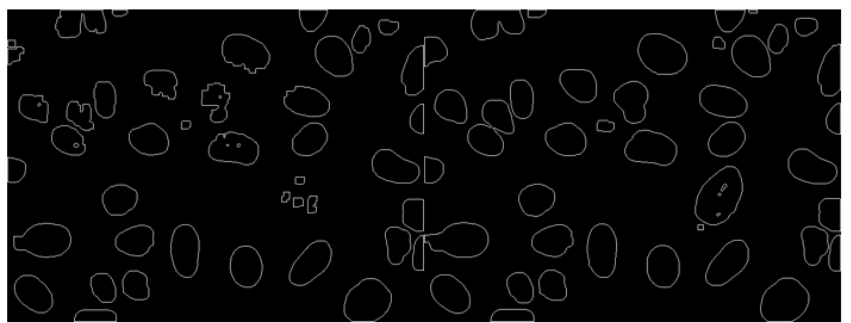

(c)

(d)

Figure 6 Edge detection of objects using (a) Mean filter (b) Median filter (c) Gaussian filter (d) MMG hybrid filter

When Figure 6 is examined, it is seen that edge detection with MMG method is more successful than mean, median and Guassian filtering methods and it also eliminates the effect of the noise in the image. Therefore, it has been determined that MMG hybrid filtering method is more successful in both edge detection and noise reduction than mean, median and Guassian filtering methods. Masked object edge detection images are given in Figure 7.

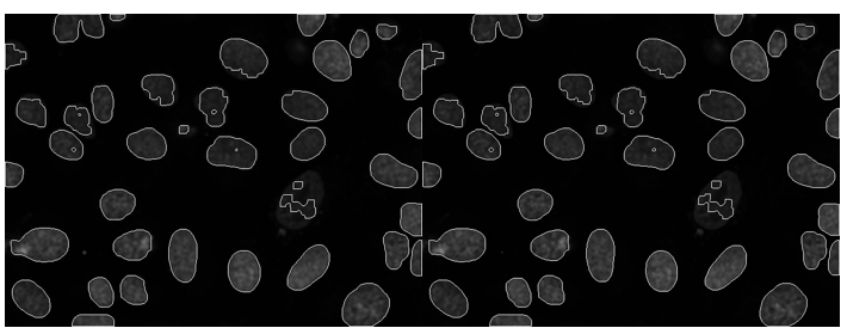

(a)

(b)

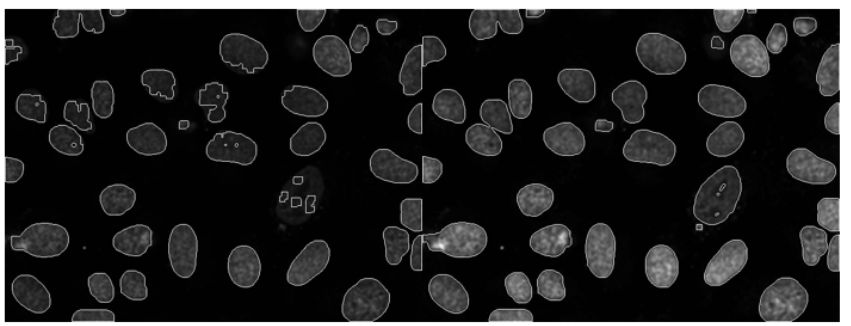

(c)

(d)

Figure 7 Masked object edge detection image of (a) Mean filter (b) Median filter (c) Gaussian filter (d) MMG filter

When Figure 7 is examined, it is seen that MMG filtering method gives more successful results than mean, median and Guassian filtering methods.

\section{Conclusions}

Today, with the rapid development of technology, image processing techniques have started to be used frequently in many fields. In this study, a new hybrid filtering method, which is an alternative to mean, median and Guassian basic image processing filtering methods, is presented. This method is based on 
the results obtained from mean, median and Guassian filtering methods. In this method, the MMG hybrid filter was created by calculating the resultant vectors of the results obtained from mean median and Guassian filters. In order to convert the created image to a 1-byte unsigned integer (uint8) format, normalization was performed by dividing it by the maximum pixel value in the image and multiplying by 255. The following results were obtained by comparing the designed MMG hybrid filter with mean, median and Guassian filtering methods for histogram and edge detection.

Firstly, in the comparison made according to histogram curves; It has been determined that MMG hybrid filter gives more suitable results than mean, median and Guassian filtering methods both on image and on histogram curves.

The second performance test of the MMG hybrid filtering method was carried out for edge detection and segmentation image processing methods. It has been observed that MMG hybrid filtering method gives more successful results in edge detection compared to mean, median and Guassian filtering methods.

It is thought that the MMG filter presented in this study will be a hybrid filtering method that can be used in the literature for more stable image softening in basic image processing and filtering methods. Also, we aimed to improve the newly developed hybrid filtering method by using different methods and optimization techniques in future academic studies.

\section{References}

[1] F. Jalled and I. Voronkov, “Object detection using image processing,” 2016. [Online]. Available: arXiv:1611.07791.

[2] S. R.Balaji and S. Karthikeyan, "A survey on moving object tracking using image processing," in Proc. 11th Int. Conf. on Intelligent Syst. and Control (ISCO), Coimbatore, India, Jan. 5-6, 2017, pp. 469-474.

[3] Q. Chen, J. Xu and V. Koltun, "Fast image processing with fully-convolutional networks," in Proc. of the IEEE Int. Conf. on Comput. Vision (ICCV), Venice, Italy, Oct. 22-29, 2017, pp. 2497-2506.

[4] G. Adlinge, S. Kashid, T. Shinde and V. Dhotre, "Text Extraction from image using MSER approach,” Int. J. Res. Eng. Technol. (IRJET), vol. 3, no. 05, 2016.

[5] P. V. Garad, "Object sorting robot based on the shape,” Int. J. of Adv. Res., Ideas and Innov. in Technol., vol. 3, pp. 129-134, 2017.

[6] G. M. Perihanoğlu "Feature Extraction From Images By Using Digital Image Processing Techniques,” M.S. thesis, Dept. Geomathic Eng., Istanbul Technical Univ., Turkey, 2015.

[7] A. M. Galal, “An analytical study on the modern history of digital photography,” Int. J. Des., vol. 26, no. 58, pp. 1-13, 2016.

[8] X. Deng, Y. Ma and M. Dong, "A new adaptive filtering method for removing salt and pepper noise based on multilayered PCNN,” Pattern Recognit. Lett., vol.79, pp. 8-17, 2016.

[9] Y. Li and S. Sun, "Research on Suppression Method of Warhead Infrared Image Background Based on Small Area Filtering,” J. Comput. Commun., vol. 6, no. 11, pp. 155-161, 2018. 
[10] W. Ma et al., "Adaptive median filtering algorithm based on divide and conquer and its application in CAPTCHA recognition,” Comput., Mater. \& Continua, vol. 58, no. 3, pp. 665-677, 2019.

[11] H. Michalak and K. Okarma, "Improvement of image binarization methods using image preprocessing with local entropy filtering for alphanumerical character recognition purposes," Entropy, vol. 21, no. 6, pp. 562, 2019.

[12] Q. Fan et al., "A generic deep architecture for single image reflection removal and image smoothing," in Proc. IEEE Int. Conf. on Comput. Vision, Venice, Italy, Oct. 22-29, 2017, pp. 32383247.

[13] W. Liu et al., "Real-time Image Smoothing via Iterative Least Squares,” 2020. [Online]. Available: arXiv:2003.07504.

[14] Q. Fan et al., "Image smoothing via unsupervised learning,” ACM Trans. on Graph. (TOG), vol. 37, no. 6, pp. 1-14, 2018.

[15] P. Li, X. Liu and H. Xiao, "Quantum image median filtering in the spatial domain,” Quantum Inf. Process., vol. 17, no. 3, pp. 49, 2018.

[16] G. George, R. M. Oommen, S. Shelly, S. S. Philipose and A. M. Varghese, "A survey on various median filtering techniques for removal of impulse noise from digital image," in Proc. 2018 Conf. on Emerg. Devices and Smart Syst. (ICEDSS), Tamilnadu, India, Mar. 2-3, 2018, pp. 235-238.

[17] P. Zhang and F. Li, "A new adaptive weighted mean filter for removing salt-and-pepper noise," IEEE Signal Process. Lett., vol. 21, no. 10, pp. 1280-1283, 2014.

[18] Z. Zhang et al., "A new adaptive switching median filter for impulse noise reduction with predetection based on evidential reasoning,” Signal Process., vol. 147, pp. 173-189, 2018.

[19] S. B. S. Fareed and S. S. Khader, "Fast adaptive and selective mean filter for the removal of highdensity salt and pepper noise,” IET Image Process., vol .12, no. 8, pp. 1378-1387, 2018.

[20] B .Gupta and S. N. Shailendra, "Image Denoising with Linear and Non-Linear Filters: A Review," Int. J. of Comput. Sci. Issues (IJCSI), vol. 10, no. 6, pp. 149-154, 2013.

[21] I. Agustina, F. Nasir and A. Setiawan, "The Implementation Of Image Smoothing To Reduce Noise Using Gaussian Filter,” Int. J. of Comput. Appl., vol. 177, no. 5, pp. 15-19, 2017.

[22] F. Cabello, J. León, Y. Iano and R. Arthur, "Implementation of a fixed-point 2D Gaussian Filter for Image Processing based on FPGA,” in Proc. 2015 Signal Process.: Algorithms, Architectures, Arrangements, and Appl. (SPA), Poznan, Poland, Sept 23-25, 2015, pp. 28-33.

[23] B. Garg and G. K. Sharma, "A quality-aware Energy-scalable Gaussian Smoothing Filter for image processing applications,” Microprocessors and Microsystems, vol. 45, pp. 1-9, 2016. 